\title{
The Association of Respiratory Syncytial Virus Infection and Childhood Asthma: A Meta-Analysis
}

\author{
Lixin Xia, Fei Yang* \\ Yangtze University Health Science Center, Jingzhou, China \\ Email:xiacelia@qq.com, ^17053717@qq.com
}

How to cite this paper: Xia, L.X. and Yang, F. (2020) The Association of Respiratory Syncytial Virus Infection and Childhood Asthma: A Meta-Analysis. Yangtze Medicine, 4, 157-162.

https://doi.org/10.4236/ym.2020.42015

Received: September 9, 2019

Accepted: June 26, 2020

Published: June 29, 2020

Copyright ( 2020 by author(s) and Scientific Research Publishing Inc. This work is licensed under the Creative Commons Attribution International License (CC BY 4.0).

http://creativecommons.org/licenses/by/4.0/

\begin{abstract}
Objective: To explore the close relationship between respiratory syncytial virus (RSV) infection and acute attack of childhood asthma. Methods: A computer-based search of database from Pumbed, CNKI, Wanfang, Baidu Scholar, Chongqing VIP, GeenMedica was performed to screen the articles about respiratory syncytial virus infection and childhood asthma. Then the literatures were screened out by the selection criteria. The RevMan5.3 software was used to test the heterogeneity and effect values of each study, analyze the sensitivity and publication bias of the literature, and draw on Meta forest plot and Funnel plot. Results: 5 articles conformed to the selection criteria. There were totally 881 cases in the case group, 826 cases in the control group. The results of heterogeneity test showed no heterogeneity between each study $(\mathrm{P}>$ 0.05). The fixed-effects model showed the 6.68 (5.06 - 8.82), $(Z=13.38, P<$ 0.00001). Conclusion: The infection rate of respiratory syncytial virus in children with acute asthma attack is higher than that in remission period. Respiratory syncytial virus infection rate can be used as an indicator of the severity of asthma in children.
\end{abstract}

\section{Keywords}

Respiratory Syncytial Virus (RSV), Asthma, Children, Meta Analysis

\section{Introduction}

The common clinical respiratory diseases in pediatrics are childhood asthma, it is also called children asthmatic bronchitis, which has a high incidence, long course and easy to repeat, and seriously affects the health of the children, even 
the safety of life [1]. The factors of asthma are divided into biologic and abiotic. Respiratory virus infection is the main cause of bronchial asthma. The study shows that the proportion of asthma in children is up to $90 \%$ [2]. Viral infection is both a source of infection and an allergen in the body, and there is also an inflammatory reaction that can cause asthma exacerbation [3]. Respiratory syncytial virus, human rhinovirus, corona virus, influenza virus and adenovirus can cause asthma attacks in children. Respiratory syncytial virus (RSV) is the most common cause of infantile wheezing, and 50\% wheezing attacks within 1 year of age [4]. The virus infection is mainly respiratory syncytial virus, the detection rate is $27.17 \%$, the detection rate of adenovirus is $3.26 \%$, and the detection rate of syncytial virus is obviously higher than that of other viruses [5]. RSV belongs to the paramyxovirus family, and is an enveloped single strand negative RNA virus [6]. The study found that the incidence of recurrent wheezing after RSV infection was higher than $60 \%$, and about $30 \%$ children were diagnosed with asthma at the age of 7.5, the high risk of asthma still existed at the age of 13 [7]. The aim of this article is to study the respiratory syncytial virus secretion during acute infection period and remission of bronchial asthma in children asthma. This article analyzed the changes of RSV infection rate in acute infection and remission stage, to further learn the clinical significance of RSV in childhood asthma.

\section{Information and Methods}

\subsection{The Sources of Data}

The data were automatically retrieved from the Pumbed, CNKI, Wanfang, Bai$\mathrm{du}$, Chongqing VIP, GeenMedica database on all cases of RSV infection and child asthma. The retrieval date was from January 2000 to March 2019, and the key words were respiratory syncytial virus and childhood asthma.

\subsection{Inclusion Criteria}

1) A random case-control study published in Chinese and English periodicals between January 2000 and March 2019;2) The incidence of RSV infection in the acute attack and remission period in childhood asthma; 3) The study was for children who less than 14 years old; 4) The required date can be obtained in the papers; 5) Eliminate some duplicate and incomplete studies.

\subsection{Selection and Extraction of Articles}

Through reading the titles and abstracts of the studies, preliminary screening is carried out, and the full text is read for secondary screening, whether the literatures are included or not is decided to the inclusion criteria. Finally, 5 articles conformed to the selection criteria, as shown in Figure 1. The relevant data of each included studies were extracted: the first author, the published years, the number of infected cases in the case group and the control group. 


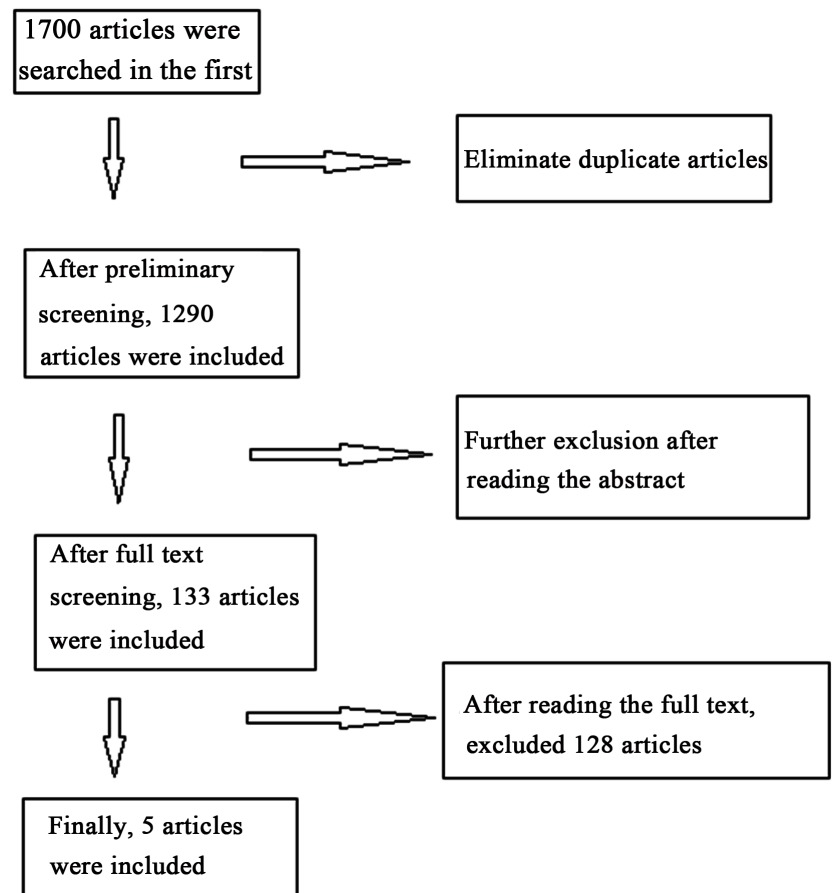

Figure 1. The literature screening process and results.

\subsection{Statistical Analysis}

RevMan5.3 software was used to analyze the whole group of 5 studies. According to the results of heterogeneity test, the corresponding analysis methods were selected. For example, there was no heterogeneity among the literatures, and the fixed effect model was used for weighted combination. If there is heterogeneity of the 5 studies, the random effect model is used for weighted combination, and finally the meta-analysis forest map and funnel map are drawn.

\section{Results}

\section{Meta-Analysis of RSV Infection in Acute Attack Stage and the Remission Period of Childhood Asthma}

We searched the articles from CNKI, Wanfang, Baidu, Chongqing VIP, Pumbed, GeenMedica database, and retrieved 17,100 related documents. After read the topics and abstracts, a total of 133 articles accorded with the topics and basic standards. After excluded the articles that could not extract data nor had incomplete data and poor article quality, 5 articles were included in Meta-analysis [8] [9] [10] [11] [12]. A total of 1707 subjects were included in the study, including 881 cases in the case group and 826 cases in the control group. The basic characteristics of the included studies are shown in Table 1.

There was no heterogeneity of all the articles $(\mathrm{P}>0.05)$, so the fixed effect model was used to carry out combined analysis. The results showed that the $95 \%$ confidence interval of the combined OR value was 6.68 (5.06 - 8.82), $(z=13.38$, $\mathrm{P}<0.00001$ ), as shown in Figure 2, which showed that the infection of RSV in acute attack stage is higher than the remission period. 


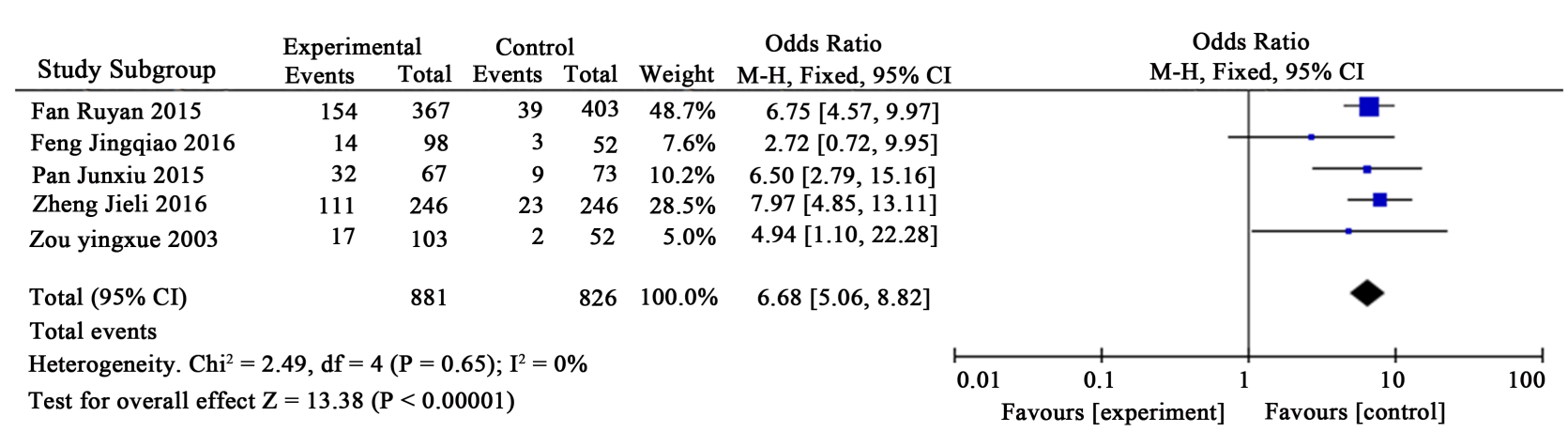

Figure 2. Forest plot of the RSV infection in acute attack stage and the remission period of childhood asthma.

Table 1. Basic features of inclusive articles.

\begin{tabular}{cccccc}
\hline Number & Author & Year & Method & $\begin{array}{c}\text { Case group } \\
(\mathrm{n} / \mathrm{N})\end{array}$ & $\begin{array}{c}\text { Control group } \\
(\mathrm{n} / \mathrm{N})\end{array}$ \\
\hline 1 & Feng Jingqiao & 2016 & Immunofluorescence & $17 / 103$ & $2 / 52$ \\
2 & Fan Ruyan & 2015 & Immunofluorescence & $154 / 367$ & $39 / 403$ \\
3 & Zheng Jieli & 2016 & Immunofluorescence & $111 / 246$ & $23 / 246$ \\
4 & Zou Yingxue & 2003 & m-RT-PCR & $17 / 103$ & $2 / 52$ \\
5 & Pan Junxiu & 2015 & Immunofluorescence & $32 / 67$ & $9 / 73$ \\
\hline
\end{tabular}

The random effect model was used to analyze 5 literatures. The OR value $(95 \% \mathrm{CI})$ was $6.72(5.09-8.86)(\mathrm{Z}=13.49, \mathrm{P}<0.00001)$, which was consistent with the results of fixed effect model, so suggesting that the results of this study are reliable.

The funnel chart was drawn using Revman5.3 software (Figure 3). This result showed that the shape is basically symmetrical and showed inverted funnel figure.

\section{Discussion}

There are reports that respiratory syncytial virus is closely related to airway hyperresponsiveness, asthma attack and recurrent wheezing. Respiratory infection is the most common cause of wheezing in children under 3 years old, accounting for $84.4 \%$ of the total wheezing cases. Respiratory syncytial virus is the main cause of wheezing related to virus infection in children with wheezing, accounting for $85.3 \%$ [13]. Some studies believe that the mechanism of recurrent wheezing caused by RSV is through stimulating the body to produce IgE, which can mediate type I allergy and cause asthma attack [14] [15], and also through destroying cilia and columnar epithelial cells on the surface of the airway, increasing permeability, causing airway hyperreactivity, enhancing airway sensitivity to allergens [16], decreasing the excitability of respiratory $\beta$ receptor or destroying cholinergic receptor, increasing the excitability of vagus nerve reaction in the airway [17].

This study explored that there is a close relationship between respiratory syncytial virus infection and acute attack of asthma in children. Through analyzing 


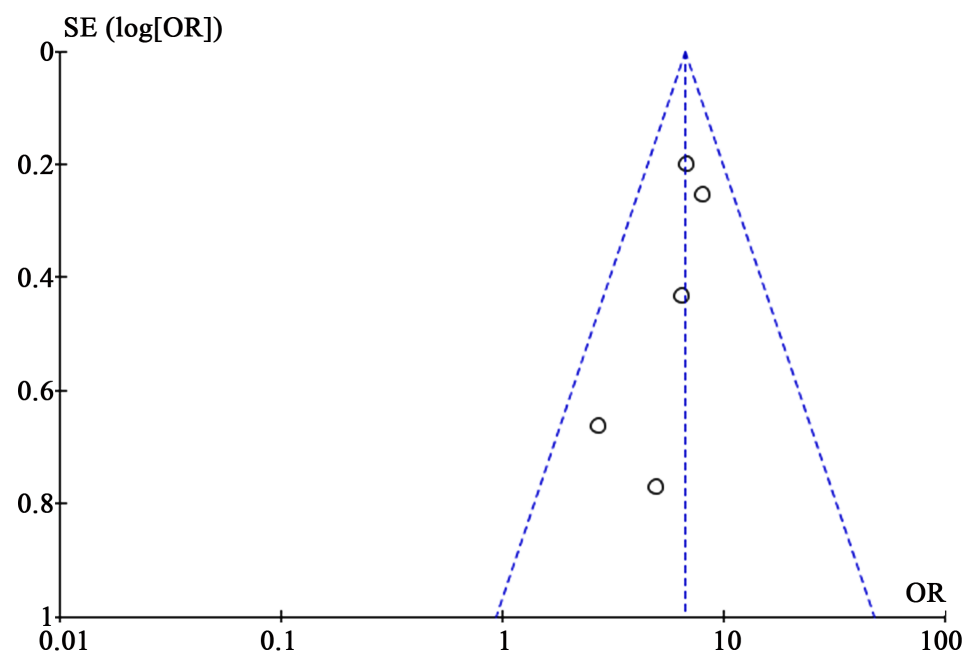

Figure 3. Funnel plot of the RSV infection in acute attack stage and the remission period of childhood asthma.

the whole group, the results showed that the infection rate in the case group is significantly higher than the control group, and the difference is highly statistically significant $(\mathrm{P}<0.00001)$. Meta-analysis results of 5 literature studies showed that RSV infection rate in the case group is higher than the control group. The $95 \%$ confidence interval of the combined OR value of each study is 6.68 (5.06 - 8.82), $(\mathrm{z}=13.38, \mathrm{P}<0.00001)$, in which the experimental group is higher than the control group of the childhood asthma. Funnel plot is basically symmetrical and has no bias.

The occurrence of childhood asthma is related to a variety of factors, such as bacterial or viral infection, air environmental impact, allergen contact, and so on. Related meta-analysis documents have counted other causes of childhood asthma, such as the correlation between asthma and vitamin $\mathrm{D}$, the relationship between gene polymorphism and asthma occurrence, the evaluation of environmental factors on asthma occurrence and so on. However, through a systematic evaluation of the literature on the respiratory syncytial virus infection in the childhood asthma, it can be preliminarily confirmed that during the acute attack of asthma, the infection rate of RSV is higher than remission period. Therefore, we think that the infection degree of RSV can be used as one of the indicators of the severity of asthma in children, and can be used to detect the clinical diagnosis and treatment. Once children's asthma is diagnosed, RSV antibody can be routine detected. Although the amount of literature included in this study is limited, this result still provides favorable evidence for clinical prevention and treatment of children's bronchial asthma, and provides a certain theoretical basis for prevention and targeted treatment of asthma.

\section{Conflicts of Interest}

The authors declare no conflicts of interest regarding the publication of this paper. 


\section{References}

[1] Zhang, K. (2014) Relationship between Asthma and Repeated Respiratory Tract Infections in Children. Journal of Clinical Pulmonary Medicine, No. 8, 1454-1456.

[2] National Cooperation Group for the Prevention and treatment of Asthma in Children (2003) A Nationwide Survey in China on Prevalence of Asthma in Urban Children. Chinese Journal of Pediatrics, No. 2, 47-51.

[3] Sang, G.M. (2010) Aminophylline Combined with Asarone for Bronchiolitis. Journal of Xinxiang Medical University, 27, 168-170.

[4] Chen, X.Y., Bao, X. and Chen, L.X. (2005) Etiological Analysis of 91 Cases of Asthma or Wheezing Attack in Children. Preventive Medicine, No. 10, 54-55.

[5] Wang, W., Chen, S.Y. and Chen, Q.R. (2016) Detection and Analysis of Respiratory tract Etiology in Children with Acute Asthma. Journal of Clinical Pulmonary Medicine, No. 10, 1907-1909.

[6] Wang, T. and Li, C.C. (2012) Relationship between Virus Infections of Respiratory Tract and Children Wheezing Diseases. Medical Recapitulate, No. 15, 2433-2435.

[7] Sigurs, N., et al. (2005) Severe Respiratory Syncytial Virus Bronchiolitis in Infancy and Asthma and Allergy at Age 13. American Journal of Respiratory and Critical Care Medicine, 171, 137-141. https://doi.org/10.1164/rccm.200406-730OC

[8] Zou, Y.Z., Li, J., Cheng, H. and Liu, L. (2003) Primary Study on the Correlation between Respiratory Viral Infection and Asthma in Children. Journal of Clinical Pediatrics, 21, 465-467.

[9] Feng, J.Q. and Lu, D.Z. (2016) Investigation of the Relationship Between the 14 Kinds of Common Respiratory Viruses and a Cute Attack of Asthma in Children in Chaoshan Area. Modern Hospitals, 16, 827-828.

[10] Fan, R.Y., Fan, C.P. and Pan, J.X. (2015) The Correlation Analysis between Virus Infection and Acute Asthmatic Attack in Children. The Journal of Medical Theory and Practice, 28, 701-703.

[11] Zheng, J.L., Zou, X.Y., Zhang, X.L. and Luo, D.Y. (2016) The Correlation Analysis between Virus Infection and Acute Asthmatic Attack in Children. Journal of Clinical Pulmonary Medicine, 21, 760-761.

[12] Pan, J.X., Fan, C.P., Fan, R.Y., et al. (2015) The Bronchoalveolar Lavage Fluid Viral Pathogens Analysis of Acute Asthmatic Attack in Children. The Journal of Medical Theory and Practice, 28, 706-708.

[13] Shen, W.-N., Wang, L. and Sun, X.-R. (2018) Retrospective Analysis of Causes of Infant Wheezing. Chinese Journal of Woman and Child Health Research, 29, 610-613.

[14] Li, L., Ren, L.H. and Ma, C. (2008) Advances of Respiratory Syncytial Virus Infection. International Journal of Immunology, 31, 295-298.

[15] Zhao, L., Mai, X., Tan, W.P., et al. (2001) Preliminary Study on Levels of IL-4, IL-12 and IgE in Children with Bronchial Asthma. Journal of Clinical Pediatrics, 19, 9-10.

[16] Khor, C.S., Sam, I.-C., Hooi, P.-S., Quek, K.-F. and Chan, Y.-F. (2012) Epidemiology and Seasonality of Respiratory Viral Infections in Hospitalized Children in Kuala Lumpur, Malaysia: A Retrospective Study of 27 Years. BMC Pediatrics, 12, 32. https://doi.org/10.1186/1471-2431-12-32

[17] Zhou, W.F., Ji, W. and Zhu, C.H. (2003) Changes and Significance of Serum TH1, TH2 Cytokines and Total IGE in Respiratory Syncytial Virus Pneumonia. Suzhou University Journal of Medical Science, 23, 211-211+215. 